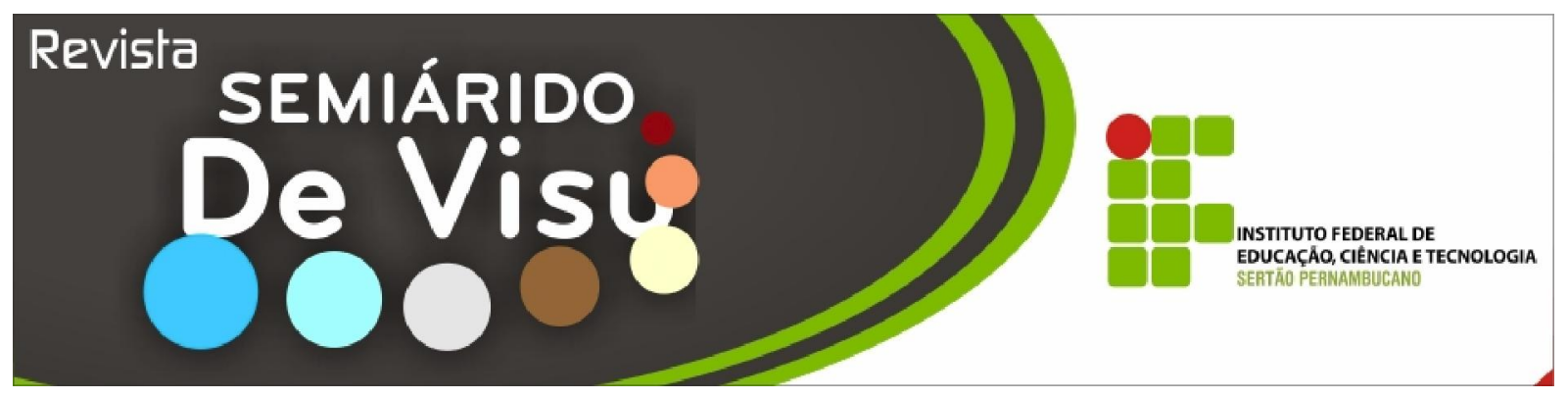

\title{
Identificação e controle preditivo do processo de craqueamento catalítico do petróleo
}

\author{
Vivianni Marques Leite dos $\operatorname{Santos}^{1}$, Maurício Bezerra de Souza $\mathrm{Jr}^{2}$ \\ ${ }^{1}$ UNIVASF, Colegiado de Engenharia de Produção, campus Juazeiro, Rodovia BA 210 Km 4 S/N CEP 48908-810 \\ Juazeiro - BA. vivianni.santos@univasf.edu.br \\ ${ }^{2}$ Escola de Química, Departamento de Engenharia Química, Sala E-207, Cidade Universitária, Ilha do Fundão, 21949- \\ 900, Rio de Janeiro-RJ. Fone: 2562-7636. mbsj@h2o.eq.ufrj.br
}

RESUMO: Redes Neurais Artificiais (RNAs) constituem uma ferramenta que vem sendo utilizada para um grande número de aplicações bem sucedidas. Sua capacidade para modelagem empírica de processos complexos vem estimulando sua aplicação em engenharia. Este artigo trata o controle do processo de craqueamento catalítico do petróleo baseado em redes neurais. Este processo é conhecido por envolver variáveis com comportamentos não lineares e com fortes interações. Para esta estratégia de controle, uma rede é usada como modelo interno para o controlador. O modelo convencional de Lee \& Kugelman (1973) foi utilizado para gerar os dados. O comportamento estacionário foi analisado através dos diagramas, de bifurcação para a concentração de coque no catalisador gasto e regenerado. A partir dos diagramas determinou-se os estados estacionários estáveis previstos para as concentrações de coque no catalisador dentro da faixa de operação. Realizou-se o controle da temperatura do riser baseado em RNAs e também o controle baseado no algoritmo DMC. Para os parâmetros ajustados, o controle baseado em RNAs mostrou mais eficiente e conservativo.

Palavras-chave: Redes Neurais Artificiais, algoritmo DMC, Processos Complexos.

\section{Identification and predictive control of the petroleum catalytic cracking process}

ABSTRACT: Artificial Neural Networks (ANNs) constitute a tool that has recently become used for a great number of successful applications. Its capacity with respect to empirical modeling of complex processes has been stimulating its application in the engineering field. This paper treats the control of the petroleum fluid catalytic cracking process based on neural networks using the multilayer kind. This process is known by its strong non-linearity and interactions between its variables. For this control strategy, a network is used as internal model for the controller. The conventional model of Lee \& Kugelman (1973) was used to obtain data. The stationary behavior was analyzed by bifurcation graphs for coke concentration in the spent and regenerated catalyst. From graphs it was determinate the stationary states expected for coke concentration in the catalyst inside the operation region. The riser temperature control based on ANNs was controlled as well as the dynamic matrix control (DMC). For the tuned parameters, the control based on ANNs was more efficient and conservative.

Key words: Artificial Neural Networks, Algorithm DMC, Complex Processes. 
Vivianni Marques Leite dos Santos

\section{Introdução}

processo de craqueamento
catalítico do petróleo em leito
fluidizado (FCC - Fluid Catalytic Cracking) tem grande importância no cenário econômico mundial, gerando cada vez mais os esforços com o objetivo de otimizar o funcionamento destas unidades. No Brasil, há grande necessidade de desenvolvimento tecnológico na área petroquímica, devido à quebra do monopólio e consequente aumento da competitividade.

Apesar da existência de diferentes técnicas de controle já estabelecidas e aplicadas satisfatoriamente a diversos processos, essas técnicas têm limitações. Foram desenvolvidas para o estudo de processos lineares ou nãolineares com regiões de operação cujas variáveis têm comportamentos lineares. Entretanto a maior parte dos processos na engenharia química são inerentemente nãolineares e em particular o processo estudado neste trabalho caracteriza-se principalmente pela natureza não linear e multivariável, com forte interação entre as variáveis manipuladas e controladas, constituindo um desafio para o controle. Nessa situação os esquemas convencionais baseados em controladores PIDs (Proporcional Integral Derivativo) independentes, podem não ser eficientes. Para processos com essas características, indica-se a utilização de técnicas avançadas de controle, como o MPC (Model Predictive Control). Esta técnica faz uso de um modelo do processo, que pode ser linear ou não linear. A estratégia DMC (Dynamic Matrix Control) vem atuando de modo bastante satisfatório. Uma classe de controladores preditivos não lineares pode ser obtida pela utilização de Redes Neurais Artificiais (RNAs). Esta estratégia é também conhecida por NNIMC (Neural Network Internal Model Control).

Neste trabalho, RNAs foram utilizadas para identificação e controle de unidades de craqueamento catalítico. Os padrões de entrada para as RNAs na etapa de treinamento, foram obtidos através do modelo fenomenológico de Lee \& Kugelman (1973). O estudo do modelo incluiu a análise do modelo linearizado e obtenção de diagramas de bifurcação. A partir do ponto de operação calculado desenvolveram duas estratégias de controle, uma baseada em RNAs e a outra no algoritmo DMC.

Devido as características peculiares do processo em estudo, neste artigo encontra-se um tópico específico para o detalhamento do mesmo.

\section{Craqueamento catalítico do petróleo e modelagem}

O processo de craqueamento do petróleo é constituído fundamentalmente por uma reação de quebra (cracking) de moléculas com alto peso molecular e baixo valor comercial, em moléculas de menor peso molecular e com alto valor comercial. O processo pode ser puramente térmico, ou pode ser realizado na presença de catalisador. Em razão do processo exigir altas temperaturas, utiliza-se o processo catalítico, que ainda assim exige temperaturas na faixa de 773 a $823 \mathrm{~K}$. A presença do catalisador também permite obtenção de maiores seletividades e portanto, maior rendimento dos produtos desejados, o que acarreta impacto significativo na indústria (Armor, 2010).

$\begin{array}{llcc}\text { A modelagem das } & \text { unidades de } \\ \text { craqueamento catalítico, necessita da } \\ \text { configuração geométrica do sistema }\end{array}$ reator/regenerador. Por sua vez, o desempenho do controlador depende do modelo adotado. O modelo desenvolvido por Lee \& Kugelman (1973) adotou o syde by syde, mostrado na Figura 1. Nesta figura pode ser identificado o riser, com menor diâmetro que o reator e logo abaixo dele, nessa unidade ocorre a maior parte das reações de craqueamento.

Sendo um processo catalítico deve-se também analisar a tendência à deposição de coque e a necessidade de unidade de regeneração do catalisador. O coque é um sólido composto por moléculas oriundas das unidades de craqueamento, que se depositam no catalisador, podendo levar a sua inativação (Araújo, 2006). 
Vivianni Marques Leite dos Santos

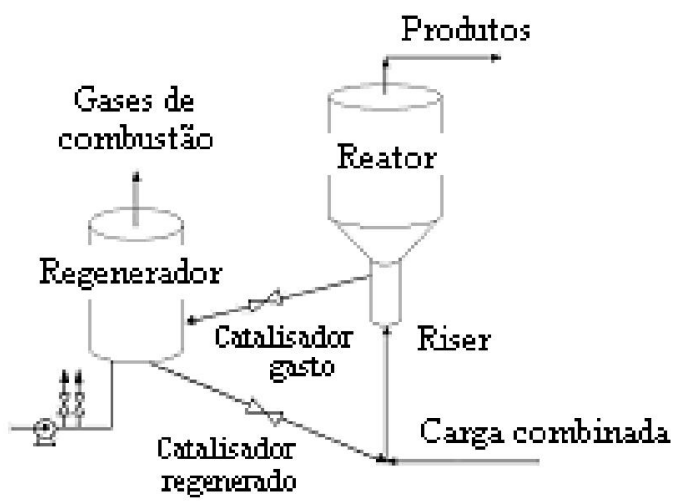

Figura 1: Modelo syde by syde da unidade de craqueamento catalítico em leito fluidizado.

O modelo é formado pelas quatro equações diferenciais ordinárias (EDOs) (Equações 1-4) a seguir:

- Balanço de carbono ou coque no reator:

$\frac{d}{d t}\left(H_{r x} C_{s c}\right)=F_{c} C_{r g}-F_{c} C_{s c}+R_{c f}$

- Balanço de energia no reator:

$\frac{d}{d t}\left(H_{r x} C_{p c} T_{r x}\right)=F_{c} C_{p c}\left(T_{r g}-T_{r x}\right)+$

$F_{t} C_{p f}\left(T_{f}-T_{r x}\right)-F_{t}\left(\lambda_{o}+\varepsilon \Delta H_{c r}\right)$

- Balanço de carbono ou coque no regenerador:

$\frac{d}{d t}\left(H_{r g} C_{r g}\right)=F_{c} C_{s c}-F_{c} C_{r g}-R_{c b}$

- Balanço de energia no regenerador:

$\frac{d}{d t}\left(H_{r g} C_{p c} T_{r g}\right)=F_{c} C_{p c}\left(T_{r x}-T_{r g}\right)+F_{a} C_{p g}\left(T_{a}-T_{r g}\right)+$

$R_{c b} Q_{c b}$

Com $\mathrm{R}_{\mathrm{cb}}, \mathrm{R}_{\mathrm{cf}}$ e $\varepsilon$ dadas por:

Taxa de queima de coque:

$$
\begin{aligned}
& R_{c b}=\frac{F_{a} M_{c}}{\alpha_{C O} M_{g}}\left(0,21-C_{O_{2}}\right) \\
& R_{c f}=a_{c c} e^{\frac{-E_{c c}}{R T_{r}}} t_{c}^{n} F_{c} \\
& \varepsilon\left(T_{r}\right)=\frac{A\left(1-e^{-\lambda}\right)}{\lambda+A\left(1-e^{-\lambda}\right)}
\end{aligned}
$$

Taxa de formação de coque:

Taxa de craqueamento:

E as seguintes quantidades intermediárias: 
Vivianni Marques Leite dos Santos

$C_{O_{2}}=0,21 e^{\left(-\frac{a_{c b} e^{\left(-\frac{E_{c b}}{R T_{r g}}\right)} C_{r g} M_{g} \alpha_{C O} H_{r g}}{0,21 F_{a} M_{c}}\right)}$
$T_{r}=0,6 T_{r x}+0,4 T_{m i x}$

Com:

$$
T_{m i x}=\frac{C_{p c} F_{c} T_{r g}+C_{p f} F_{t} T_{f}-F_{t} \lambda_{0}}{C_{p c} F_{c}+C_{p f} F_{t}}
$$

$A=C_{1} e^{\frac{Q_{1}}{R T_{r}}}$

$t_{c}=\frac{H_{r x}}{F_{c}}$

$\lambda=t_{c} C_{2} e^{-\frac{Q_{3}}{R T_{r}}}$ calculados:

A Tabela 1 mostra os valores dos parâmetros do FCC para os estados estacionários

Tabela 1 - Valores das variáveis de entrada e dos parâmetros do FCC.

$\begin{array}{ll}\mathrm{a}_{\mathrm{cb}}=3,9 \times 10^{7} \mathrm{~s}^{-1} & \mathrm{a}_{\mathrm{cc}}=0,0195 \mathrm{~kg} \text { coque } / \mathrm{kg} \text { catalisador } \\ \mathrm{C}_{1}=6990 & \mathrm{C}_{2}=111,1 \\ \mathrm{C}_{\mathrm{pc}}=2,54 \mathrm{~J} / \mathrm{kg} \mathrm{K} & \mathrm{C}_{\mathrm{pf}}=6,80 \mathrm{~J} / \mathrm{kg} \mathrm{K} \\ \mathrm{C}_{\mathrm{pg}}=0,2405 & \mathrm{E}_{\mathrm{cb}}=1,57 \times 10^{5} \mathrm{~J} / \mathrm{mol} \\ \mathrm{E}_{\mathrm{cc}}=1,02 \times 10^{4} \mathrm{~J} / \mathrm{mol} & \mathrm{F}_{\mathrm{t}}=113,02 \mathrm{~kg} / \mathrm{s} \\ \mathrm{H}_{\mathrm{rg}}=1,82 \times 10^{5} \mathrm{~kg} & \mathrm{H}_{\mathrm{rx}}=4,54 \times 10^{4} \mathrm{lb} \\ \mathrm{M}_{\mathrm{c}}=13 \mathrm{~kg} / \mathrm{kgmol} & \mathrm{M}_{\mathrm{g}}=29,2 \mathrm{~kg} / \mathrm{kgmol} \\ \mathrm{n}=-0,07 & \mathrm{Q}_{1}=2,4 \times 10^{3} \mathrm{~kJ} / \mathrm{kgmol} \\ \mathrm{Q}_{3}=-4,06 \times 10^{2} \mathrm{~kJ} / \mathrm{kgmol} & \mathrm{Q}_{\mathrm{cb}}=3,18 \times 10^{7} \mathrm{~J} / \mathrm{kg} \\ \mathrm{R}=8,31 \mathrm{~J} / \mathrm{mol} \mathrm{K} & \mathrm{T}_{\mathrm{a}}=305,15 \mathrm{~K} \\ \mathrm{~T}_{\mathrm{f}}=499,15 \mathrm{~K} & \alpha_{\mathrm{CO}}=1 \\ \Delta \mathrm{H}_{\mathrm{cr}}=2,34 \times 10^{5} \mathrm{~J} / \mathrm{kg} & \lambda_{\mathrm{o}}=2,21 \times 10^{5} \mathrm{~J} / \mathrm{kg}\end{array}$

Este modelo tem como variáveis de estado, as concentrações de coque no catalisador regenerado e no catalisador gasto e as temperaturas do riser e reator. Dentre elas, foi escolhida a temperatura do riser como variável de controle, devido à facilidade de medida e ao fato de que no riser ocorre a maior parte das reações de craqueamento, estando, portanto, diretamente relacionada à determinação do rendimento dos produtos. Por isso, na etapa de identificação, deu-se especial importância ao comportamento desta variável.

\section{Redes neurais artificiais}

Para identificação e controle foram utilizadas redes neurais artificiais - RNA. Uma RNA é formada por uma densa rede de neurônios ou elementos de processamento interconectados, que operam paralelamente, operando informações a partir de entradas, para obtenção de uma resposta dinâmica. A função de ativação sigmoidal (equação 13) tem sido aplicada com sucesso (Rumelhart e McClelland, 1986), sendo adotada neste trabalho. 
Vivianni Marques Leite dos Santos

$$
f\left(x_{i}\right)=\frac{1}{\left(1+e^{-x i}\right)}
$$

A relação entre a entrada e a saída da rede é desenvolvida na fase denominada de aprendizagem ou de treinamento. Neste trabalho utilizou-se o método de treinamento do tipo "supervisionado", durante o qual a entrada e saída são fornecidas à RNA, definindo qual o comportamento deverá desenvolver, ajustando os pesos e biases de modo a obter a saída desejada. O aprendizado supervisionado é também chamado de aprendizado por correção de erro (ou ErrorCorrection Learning) é também o método mais utilizado.

Adotou-se o método backpropagation de treinamento, minimizando a função objetivo através da técnica de gradiente conjugado (Leonard e Kramer, 1990). O método backpropagation ocorre em duas etapas, na primeira os padrões são apresentados a RNA e propagadas até que a saída seja calculada, quando são comparadas com as saídas desejadas e calculado o erro, em seguida o erro obtido na primeira etapa é propagado no sentido contrário, ajustando os pesos e biases.

O desempenho do controle baseado em redes neurais foi comparado com o controle MPC-Model Predictive Control, descrito em tópico a seguir.

\section{Controle preditivo}

O MPC - (Model Predictive Control) é uma técnica de controle avançada com várias aplicações reportadas na literatura (Stewart B. T. et al. 2010; Scattolini, 2009; AlGhazzawi \& Lennox, 2009; Daraoui et al., 2010). Sua aplicação ao processo FCC deve-se principalmente ao fato de que esses controladores podem atuar eficientemente em processos multivariáveis, com restrições nas suas variáveis.

A estratégia de controle baseia-se em informações passadas de entrada u e de saída y e numa projeção para as ações futuras de controle. A saída de um modelo matemático (chamado modelo interno), que representa o processo, é predita ao longo de um horizonte de tempos de amostragem $\mathrm{H}$ (conhecido como horizonte de predição). Uma sequência de setpoints futuros $\mathrm{r}$ é gerada, chamada trajetória de referência. Uma função objetivo apropriada de erros futuros e ações de controle é minimizada para fornecer uma sequência de ações de controle futuras. As ações de controle são selecionadas e a resposta predita apresenta determinadas características desejadas, sendo que estas ações somente podem variar dentro do horizonte $\mathrm{U} \leq \mathrm{H}$, conhecido como horizonte de controle. Apenas a primeira ação de controle calculada é implementada no processo real, para a qual as variáveis controladas são medidas. No próximo tempo de amostragem, o mesmo problema de otimização é resolvido com a nova condição inicial obtida mediante informações tiradas da planta neste último instante, com o horizonte de predição movido adiante por um tempo de amostragem. Sendo assim, a técnica ficou conhecida como de horizonte móvel (moving horizon ou receding horizon control).

A ideia central em um controle preditivo é o uso de horizontes, além dos horizontes U e $\mathrm{H}$, tem-se ainda o horizonte do modelo $\mathrm{T}$, que é o tempo, para a resposta do passo em laço aberto, está $99 \%$ completa. Está associado com o tempo de estabilização do sistema em malha aberta e ao período de amostragem adotado (Fileti, 1998).

Os controladores MPC podem ser diferenciados em função do modelo interno. Este modelo pode ser linear ou não-linear, dependendo do problema a ser tratado. As primeiras versões de controladores preditivos eram baseadas em modelos lineares. Controladores preditivos não-lineares são obtidos, utilizando-se modelos internos não lineares, como por exemplo uma RNA.

A estratégia de controle foi desenvolvida, usando RNAs e também o algoritmo DMC (Dynamic Matrix Control). Para este último, utiliza-se o modelo não 
Vivianni Marques Leite dos Santos

paramétrico de convolução discreta da resposta ao degrau. $\mathrm{O}$ modelo fenomenológico foi utilizado para gerar dados na identificação do processo pela RNA que, após a identificação, substituiu o modelo na estratégia de controle.

\section{Materiais e métodos}

Para o desenvolvimento deste trabalho, foi utilizado o modelo de Lee \& Kugelman, 1973 para gerar dados do treinamento das RNAs, de modo que, este representou o próprio processo. Os programas foram desenvolvidos em linguagem FORTRAN, tanto para a identificação e controle baseado em RNAs como para o desenvolvimento da estratégia DMC.
Um Estado Estacionário (EE) foi inicialmente obtido, usando o método de Newton-Raphson, implementado no MapleVRelease5. Realizou-se um estudo dos estados estacionários previstos pelo modelo, utilizando o estado estacionário calculado, inicialmente como entrada para o AUTO97 (Doedel et al., 1998), software que permitiu a obtenção de bifurcações dinâmicas para as equações diferenciais ordinárias - EDOs.

Narendra \& Parthasaraty (1990), sugeriram quatro classes de modelos para processos não-lineares, os quais são descritos por equações de diferenças finitas. O mais geral e, portanto com maior dificuldade de implementação, é mostrado a seguir (equação 14) escolhida para este estudo.

$$
y(k+1)=f[y(k), y(k-1), \ldots, y(k-n+1) ; u(k), u(k-1), \ldots, u(k-m+1)
$$

Nesta equação, f e g são funções nãolineares quaisquer, $\mathrm{m}$ e $\mathrm{n}$, estão relacionados à ordem do sistema e devem ser adotados com base na análise dos dados do processo, conhecimento prévio sobre o mesmo e experimentação. $\quad \mathrm{u}(\mathrm{k})$ e $\mathrm{y}(\mathrm{k})$ representam respectivamente, a entrada e a saída de um processo SISO no tempo de amostragem $\mathrm{k}$.

Narendra e Parthasathy (1990) também propõem que para a identificação de um processo, dois métodos são possíveis: método série-paralelo (mps) e método paralelo (mp). No primeiro, a saída do processo é alimentada na rede neural. Ao passo que, no último, as saídas atrasadas da própria rede são alimentadas à mesma, e a Equação 14, passa a ser escrita na forma:

$$
\widehat{y}(k+1)=\hat{f}\left[\begin{array}{l}
\hat{y}(k), \hat{y}(k-1), \ldots, \widehat{y}(k-n+1) ; \\
u(k), u(k-1), \ldots, u(k-m+1)
\end{array}\right]
$$

No método backpropagation de treinamento, os sinais para trás (redes recorrentes) não estão presentes, permitindo apenas o treinamento no esquema sérieparalelo. Existem trabalhos onde se usou uma modificação do método backpropagation de modo a incluir recorrência (SU et al., 1992).

As RNAs foram treinadas, adotando o esquema série-paralelo para predições de um passo adiante e usadas como as redes paralelas em predição dos estados estacionários.

\section{Resultados e discussão}

\section{Estudo do modelo em malha aberta - Comportamento estacionário}

A análise da estabilidade dos pontos estacionários foi realizada linearizando o modelo e obtendo suas funções de transferência. Para o ponto cuja vazão de ar é igual a $7,4117 \times 10^{5}$ (Tabela 2 ), as raízes do polinômio do denominador apresentaram parte real negativa, uma característica de um ponto 
Vivianni Marques Leite dos Santos

estável. Este ponto foi escolhido para estudos posteriores mais detalhados.

Tabela 2 - Variáveis de estado e vazões de entrada para o Modelo baseado em redes neurais artificiais.

\begin{tabular}{ll}
\hline $\operatorname{Trg}=1354,3^{\circ} \mathrm{F}(1007,8 \mathrm{~K})$ & $\mathrm{Fc}=1081,64 \mathrm{~kg} / \mathrm{s}$ \\
$\operatorname{Tr} \mathrm{x}=1126,6^{\circ} \mathrm{F}(881,3 \mathrm{~K})$ & $\mathrm{Fa}=93,39 \mathrm{~kg} / \mathrm{s}$ \\
$\mathrm{Csc}=6,87 \times 10^{-3} \mathrm{~kg}$ coque $/ \mathrm{kg}$ catalis. & $\mathrm{Crg}=2,99 \times 10^{-4} \mathrm{~kg}$ coque $/ \mathrm{kg}$ catalis. \\
\hline
\end{tabular}

Os resultados para o estudo dos estados estacionários foram apresentados na forma de ramos de soluções estáveis (linha sólida) e instáveis (linha tracejada) e também um ponto de bifurcação de Hopf, que consiste na bifurcação de um equilíbrio para uma oscilação periódica (Fiedler-Ferrara \& Prado, 1995). Dessa forma, obteve-se estados estacionários estáveis previstos para as concentrações de coque no catalisador dentro da faixa de operação, estão restritos a faixa de vazão de ar $83,75-108,23 \mathrm{~kg} / \mathrm{s}$, como mostra a Figura 2.

Para uma vazão de ar igual a 108,23 $\mathrm{kg} / \mathrm{s}$ existe um pequeno ramo de soluções oscilatórias estáveis (círculos cheios), que podem ser melhor visualizadas na Figura 3.

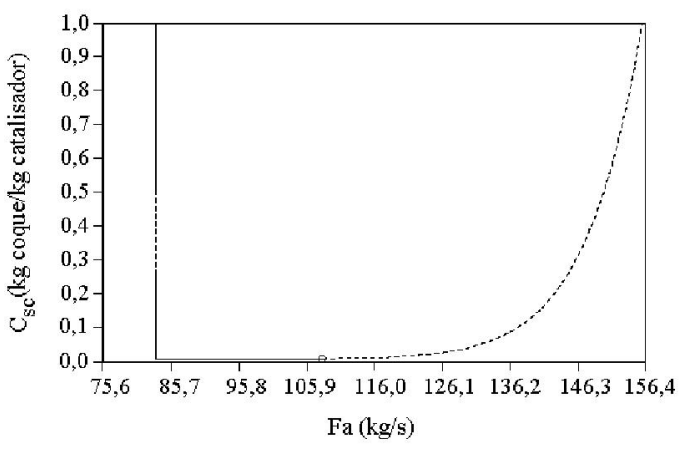

(a)

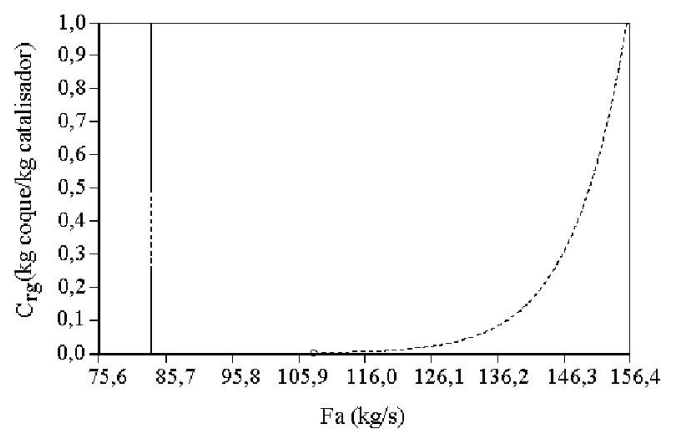

(b)

Figura 2: Bifurcação dinâmica para a concentração de coque no catalisador gasto (a) e para a concentração de coque no catalisador regenerado (b).

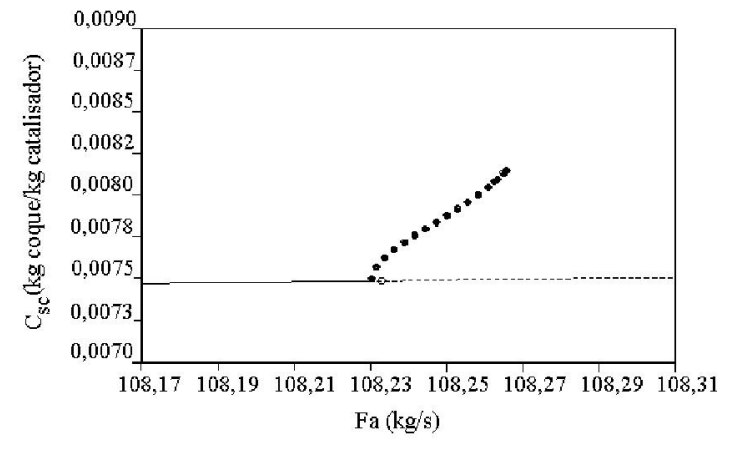

(a)

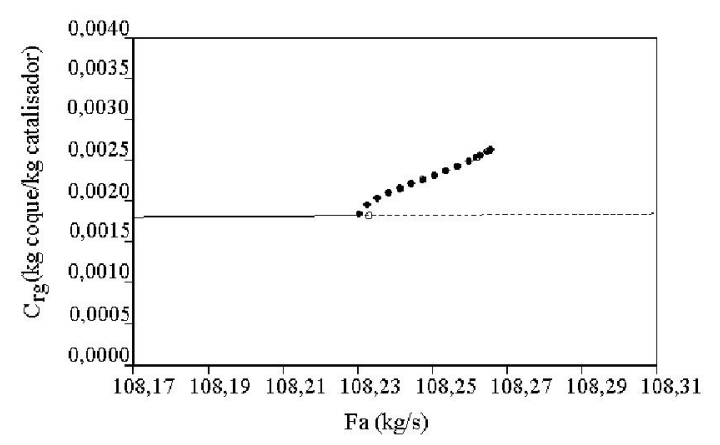

(b)

Figura 3: Bifurcação dinâmica para a concentração de coque no catalisador gasto (a) e no catalisador regenerado (b) para a faixa $108,17-108,31 \mathrm{~kg} / \mathrm{s}$. 
Como pode ser observado nas Figuras 2 e 3, o ponto estacionário escolhido (Tabela 2) está dentro desta faixa e satisfaz às restrições do processo, por esta razão, foi mantido este ponto operacional na etapa de controle.

\section{Estudo do modelo em malha aberta - Comportamento dinâmico}

Os resultados para o modelo syde by syde, cujas equações diferenciais ordinárias (EDOs) foram linearizadas, mostraram que para pequenas mudanças operacionais, ocorre predição do comportamento com acurácia, mas para degraus maiores, a não linearidade é

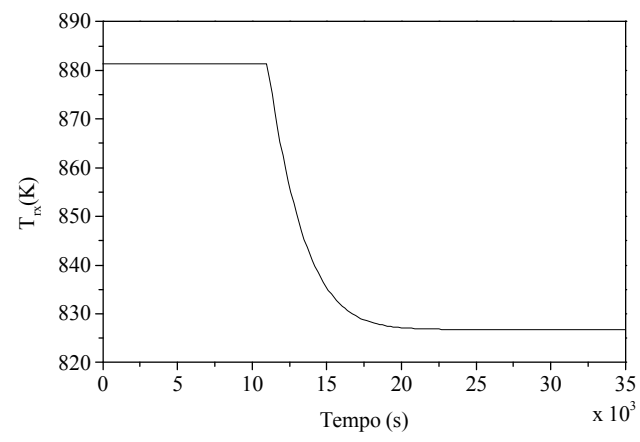

(a) evidente, de modo que, as curvas dadas pelos modelos não-linear e linearizado se distanciam significativamente.

A simulação dinâmica do modelo não linear prediz o comportamento da temperatura do riser, para a resposta a um degrau de $10,0 \%$ na vazão de ar para o regenerador $(93,39$ para $102,72 \mathrm{~kg} / \mathrm{s}$ ) como revela na Figura 4 (a) a seguir.

A constante de tempo $\left(\tau_{\mathrm{a}}\right)$ foi obtida, sendo aproximadamente igual a $1,0 \mathrm{~h}$ ou 60,0 min, que foi utilizado para determinação do tempo de amostragem $\left(\mathrm{Ta}=0,05 \mathrm{~h}\right.$ ou $\left.5 \% \mathrm{x} \tau_{\mathrm{a}}\right)$. O comportamento das demais variáveis de estado são mostrados nas Figuras 4 (b) e 5.

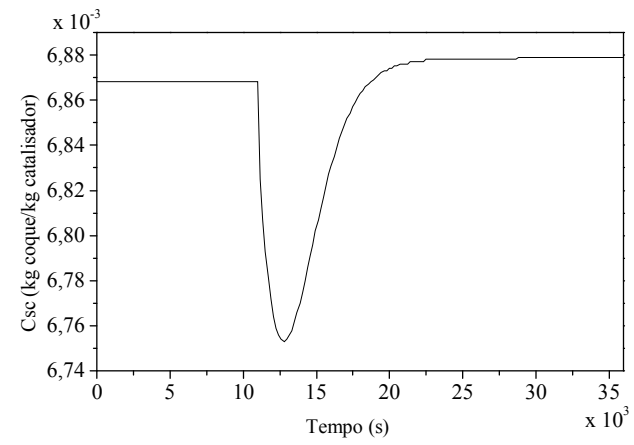

(b)

Figura 4: Comportamento da temperatura do riser (esquerda) e da concentração de coque no catalisador gasto (direita) devido a um estímulo degrau igual a $9,33 \mathrm{~kg} / \mathrm{s}(10,0 \%)$ na vazão de ar para o regenerador.

O degrau na vazão de ar foi aumentado sucessivamente até que, para um degrau de $16,0 \%(\mathrm{Fa}=108,33 \mathrm{~kg} / \mathrm{s})$ o sistema se tornou

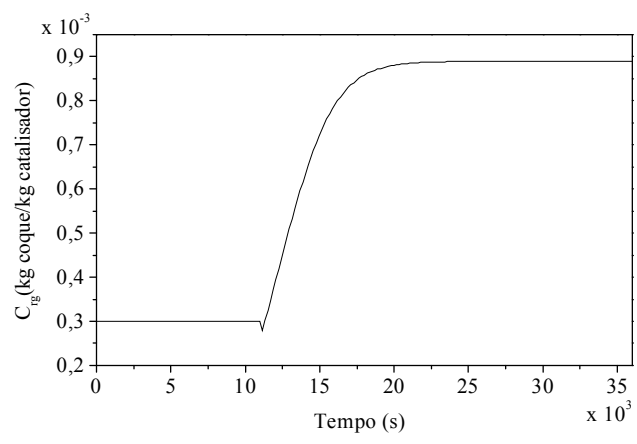

(a) instável para a concentração de coque, como pode ser observado na Figura 6 (a).

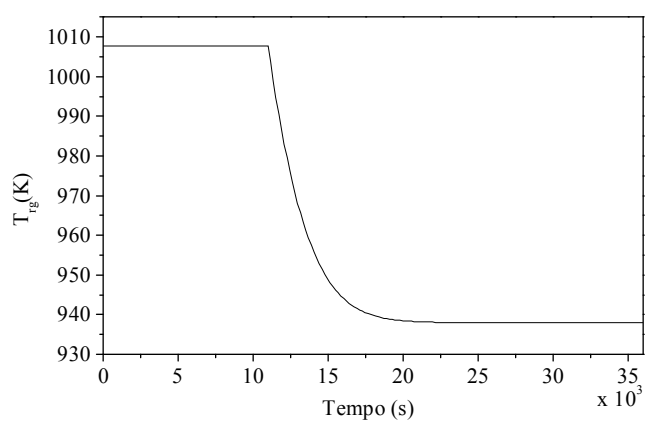

(b)

Figura 5: Comportamento da concentração de coque no catalisador regenerado (a) e da temperatura do regenerador (b), devido a um estímulo degrau de $10,0 \%$ na vazão de ar para o regenerador. 
Vivianni Marques Leite dos Santos

Diminuindo a vazão de ar, após um degrau de $-11,0 \%(83,12 \mathrm{~kg} / \mathrm{s})$ o sistema teve comportamento semelhante. A partir destes pontos as concentrações de coque tendem a um estado estacionário distante do ponto de operação. Estas conclusões estão de acordo com os resultados fornecidos pelo AUTO97 (Doedel et al., 1998).

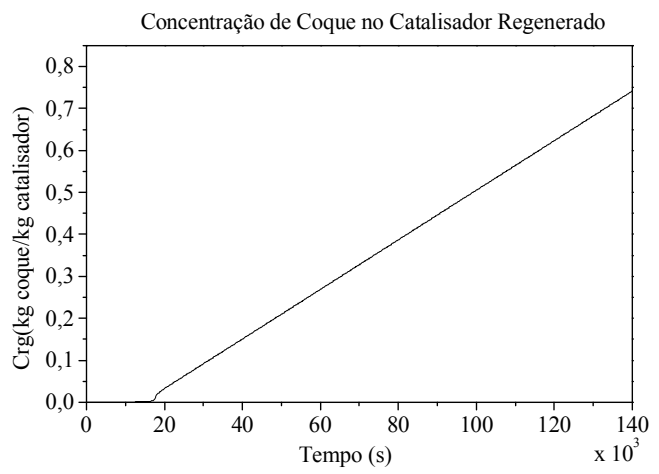

(a)

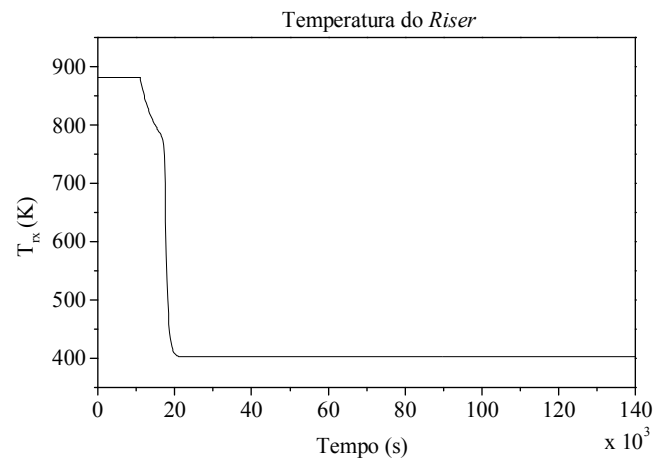

(b)

Figura 6: Comportamento da concentração de coque no catalisador regenerado (a) e da temperatura do riser (b) devido a um estímulo degrau igual a 108,33 kg/s $(16,0 \%)$ na vazão de ar para o regenerador.

O comportamento observado nos degraus acima de $16,0 \%$ pode ser explicado pela análise do comportamento estacionário do processo para a temperatura do regenerador. Como se pode notar, esta temperatura atinge um estado estacionário próximo de $366,5 \mathrm{~K}$. Isto faz com que a concentração de coque aumente muito.

\section{Resultados do treinamento das RNAs}

O programa elaborado para simulação

$$
y_{\text {escala }}=0,1+\left[\frac{\left(y-y_{\min }\right)}{\left(y_{\max }-y_{\min }\right)}\right] x 0,8
$$

Foi utilizada a função de ativação sigmoidal, com 2 neurônios na camada de entrada, os quais recebem dados da vazão de ar para o regenerador e da temperatura do riser no tempo de amostragem atual (multiple input), e um neurônio na camada de saída (single output), com a temperatura do riser no tempo gerou 1600 dados, dos quais 800 foram utilizados para treinar as RNAs e 800, para testá-las, na faixa $-20,0 \%+15,0 \%$ (ou 74,71 $107,39 \mathrm{~kg} / \mathrm{s}$ ), a partir do estado estacionário inicial $(93,39 \mathrm{~kg} / \mathrm{s})$. O uso da função de ativação sigmoidal restringe a saída da rede entre 0 e 1,0. Por isso, os dados de treinamento deveriam ser normalizados nesta faixa. Entretanto, optou-se pela normalização dos dados entre 0,1 e 0,9 (Equação 16), evitando assim a ocorrência de pesos muito grandes e permitisse eventuais extrapolações. de amostragem seguinte. $O$ número de neurônios na camada escondida $(\mathrm{Nh})$ foi determinado, variando-se o número de neurônios de 1 a 6 . Com apenas 1 neurônio na camada escondida, não foi possível convergência. Os resultados obtidos para os demais casos estão resumidos na Tabela 3. Os 
Vivianni Marques Leite dos Santos

valores entre parênteses são dados na forma ( $\mathrm{Ni}, \mathrm{Nh}, \mathrm{No}$ ), onde $\mathrm{Ni}$, Nh e No representam o número de neurônios nas camadas de entrada, escondida e de saída, respectivamente.

Utilizou-se o método do gradiente conjugado, dentre suas vantagens, o mesmo considera não apenas a direção descendente, tal como o método do gradiente descendente, mas também a curvatura da superfície, sendo portanto, mais rápido que o método do gradiente descendente.

Para determinar qual RNA produz a melhor generalização, a partir dos mesmos dados de treinamento, usou-se inicialmente, o método da validação cruzada. Neste método, os dados da base histórica são divididos em duas partes, uma utilizada para treinamento da rede (padrões de treinamento) e a outra para testar a capacidade de generalização da rede (padrões de teste).

Tabela 3 - Resultados do treinamento das RNAs, com apresentação da função objetivo, gradiente, número de interações necessárias e o número de pesos e biases que formam a rede neural obtida.

\begin{tabular}{ccccc}
\hline RNA & Função Objetivo & Gradiente & No Iterações & $\begin{array}{c}\text { No Pesos e } \\
\text { Biases }\end{array}$ \\
\hline$(2: 2: 1)$ & $1,296 \times 10^{-2}$ & $7,762 \times 10^{-9}$ & 491 & 9 \\
$(2: 3: 1)$ & $1,003 \times 10^{-2}$ & $9,109 \times 10^{-9}$ & 994 & 13 \\
$(2: 4: 1)$ & $7,394 \times 10^{-3}$ & $8,203 \times 10^{-9}$ & 2234 & 17 \\
$(2: 5: 1)$ & $7,238 \times 10^{-3}$ & $9,357 \times 10^{-9}$ & 2693 & 21 \\
$(2: 6: 1)$ & $7,225 \times 10^{-3}$ & $3,923 \times 10^{-9}$ & 2459 & 25 \\
\hline
\end{tabular}

Analisando os dados de treinamento e de teste, nota-se que apesar do uso de degraus dentro da mesma faixa para ambos, atingiu-se um ponto de máxima maior para os dados de teste e valor de temperatura fora da faixa recebida pela rede no treinamento. A predição deste ponto foi possível devido à normalização adotada, que permite pequenas extrapolações. $\mathrm{O}$ valor máximo na etapa de treinamento foi de $1359,9^{\circ} \mathrm{F}$, e $1384,2^{\circ} \mathrm{F}$ para os dados de teste.

Os resultados dos testes foram muito bons para todas as redes treinadas. Sendo que, a rede com $\mathrm{Nh}=5$ permitiu melhor generalização para o ponto de máximo (erro menor e igual a $0,65 \%)$.

Para promover a escolha da melhor RNA, além do método da validação cruzada, adotou-se também um método menos conservativo e desafiador, que é a predição da saída constante (estado estacionário - EE) pela
RNA, a partir dos EEs iniciais e daqueles atingidos a cada incremento de vazão.

As RNAs com 4, 5 e 6 neurônios na camada escondida conduziram a resultados melhores que aquelas com 2 e 3 neurônios, prevendo os EEs em maior concordância com os dados do modelo. Entretanto, para a RNA com 6 neurônios na camada escondida, os estados estacionários se distanciam daqueles previstos pelo modelo, ou seja, a rede começa a perder sua capacidade de generalização. Isto pode ser verificado nas Figuras a seguir, que ilustram os EEs previstos pelo modelo (implementado em Fortran90) e pelas RNAs, com Nh igual a 4, 5 e 6 (Figura 7), a partir do EE inicial, e a Figura 8, que mostra os EEs atingidos a partir do EE a cada incremento da vazão, com a vazão de ar aumentando ou diminuindo como indicado pelas setas. 
Vivianni Marques Leite dos Santos

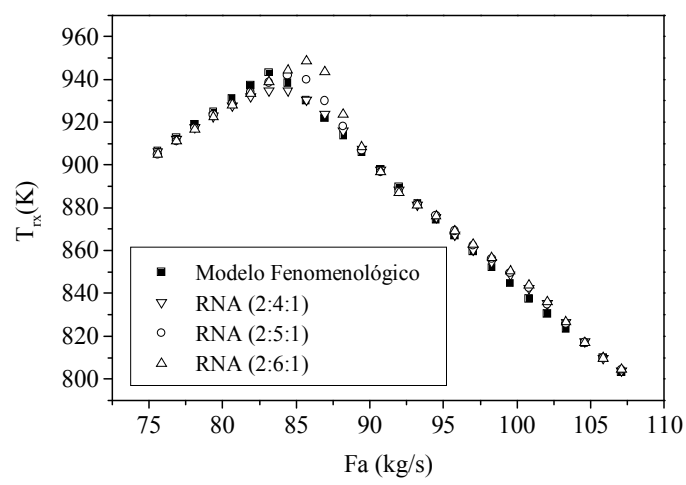

Figura 7: EEs previstos pelo modelo e pelas RNAs $(2: 4: 1),(2: 5: 1)$ e $(2: 6: 1)$, a partir dos estados estacionários iniciais.

A definição do número de neurônios na camada escondida é determinada nesta etapa de validação, devendo-se observar neste ponto que um grande número de neurônios não determina os resultados serão melhores e na maior parte

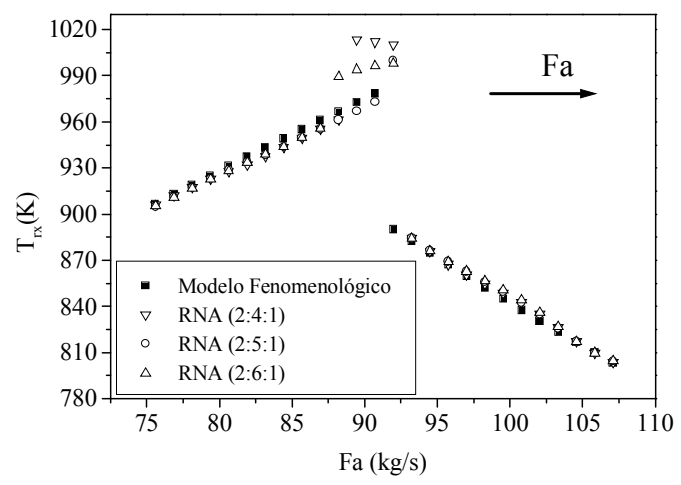

(a) das vezes leva à perda de capacidade de generalização da rede neural, como pode ser observado neste estudo o fato, que a rede com maior número, neste caso 6 neurônios, levou a previsão de EEs com menor exatidão.

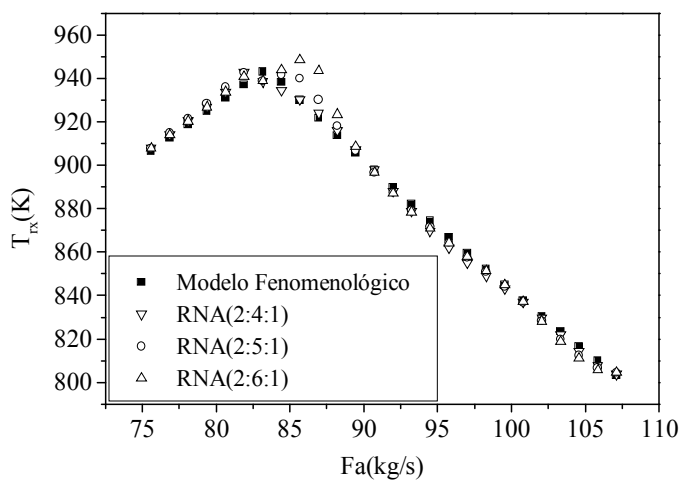

(b)

Figura 8: EEs previstos pelo modelo e pelas RNAs $(2: 4: 1)$, (2:5:1) e (2:6:1), a partir dos EEs atingidos a cada aumento da vazão (a) e a cada redução da vazão (b).

A análise das Figuras 8 - (a) e 8 - (b) e os resultados mostrados na Tabela 3 indicam melhores resultados, utilizando-se 5 neurônios na camada escondida. A estrutura da RNA feedforward final para predição da temperatura do riser é dada por (2:5:1), ou seja, dois, cinco e um neurônio nas camadas de entrada, escondida e de saída, respectivamente.

Após a definição da rede neural artificial esta foi utilizada para uma das propostas de controle foco deste trabalho. Os resultados obtidos para o controle, usando redes e o algoritmo DMC são apresentados a seguir em tópico específico.

\section{Controle Preditivo MISO usando RNAs e usando o Algoritmo DMC}

A estratégia de controle baseada em RNAs é ilustrada na Figura 9 a seguir: 
Vivianni Marques Leite dos Santos

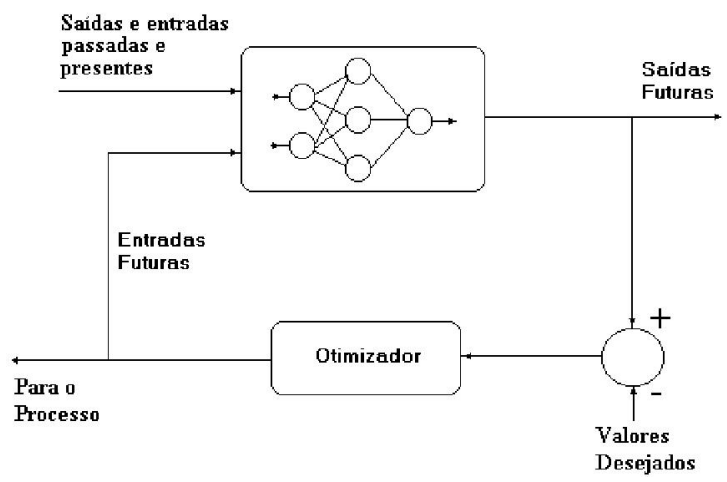

Figura 9: Esquema do controle preditivo usando RNAs.

Como podemos observar na Figura 9, a rede neural é capaz de prever variáveis dependentes do tempo, recebendo saídas e entradas (passadas e presentes), gerando saídas futuras. As saídas futuras são comparadas com valores desejados através do otimizador, cuja ação de controle será conduzida para o processo.

Depois da identificação, a RNA está apta para o controle, fazendo predições um passo adiante, em um total de $\mathrm{H}$ predições no futuro.

A minimização da função objetivo (equação 17) foi realizada, empregando a rotina DNCONF, da biblioteca IMSL, interna ao Microsoft Fortran PowerStation 4.0. Esta rotina implementa o método de programação quadrática sucessiva, mas não está disponível na forma aberta, e portanto não se tem acesso aos programas fontes.

$$
J(k)=\sum_{i=1}^{H}\left[\hat{y}(k+i)-y_{s p}(k+i)\right]^{2}+\lambda \sum_{i=1}^{U}[\Delta u(k+i-1)]^{2}
$$

Em que: $u(k+i)=u(k+U-1), i=U, \ldots, H \mathrm{e}$ $\lambda$ é o fator de ponderação nos incrementos das ações de controle calculadas.

$$
\begin{gathered}
y_{\text {min }} \leq \hat{y}(k+i) \leq y_{\text {mix }}, i=1, \ldots, H \\
u_{\text {min }} \leq u(k+i-1) \leq u_{\text {max }}, i=1, \ldots, H \\
{[u(k+i)-u(k+i-1)] \leq \Delta u_{\max }, i=1, \ldots, U}
\end{gathered}
$$

A trajetória de referência para a estratégia de controle é dada pelo modelo:

$$
\begin{gathered}
y_{s p}(k)=y(k) \\
y_{s p}^{(k+i)}=\alpha y_{s p}^{k+i-1}+(1-\alpha) s p
\end{gathered}
$$

Cada predição de um passo adiante da correção (De Souza Jr, 1993) dada por: RNA foi corrigida, usando uma variável de

$$
d(k)=y(k)-\hat{y}(k \mid k-1)
$$

A predição corrigida então fica: 
Vivianni Marques Leite dos Santos

$$
\hat{y}_{d}(k+1 \mid k)=\hat{y}(k+1 \mid k)+d(k)
$$

Em que: $\mathrm{y}(\mathrm{k})$ é a saída real do processo no tempo de amostragem $\mathrm{k}, \hat{y}(k \mid k-1)$, a predição da rede neural no tempo de amostragem anterior e $\hat{y}_{d}(k \mid k+1)$ é a variável corrigida.

No algoritmo DMC as ações de controle

$$
\begin{aligned}
& J(\Delta u)=\Delta \underline{u}^{T} \cdot \underline{A}^{T} \cdot \underline{A} \cdot \Delta \underline{u}-\Delta \underline{u}^{T} \cdot \underline{A}^{T} \cdot \hat{E}^{\prime \prime}- \\
& \underline{E}^{\prime T} \cdot \underline{\underline{A}} \cdot \Delta \underline{u}+\underline{E}^{\prime T} \cdot \underline{\underline{E}}
\end{aligned}
$$

são obtidas mediante a minimização da equação (25) a seguir, dada pelo somatório do quadrado das diferenças entre as variáveis desejadas $\left(\mathrm{y}^{\mathrm{d}}\right)$ e as predições corrigidas $\left(\mathrm{y}^{\mathrm{C}}\right)$, para todos os $\mathrm{H}$ instantes de predição.
Em que: $\stackrel{A}{=}$ é a matrix dinâmica, $\Delta \underline{u}^{T}$ é o vetor de ações de controle, e $\underline{\underline{E}}^{\prime}$ vetor de erros ponderados por $\alpha$, um parâmetro de suavização da ação de controle. das variáveis de saída $(\widehat{E})$ e da diferença entre as ações de controle consecutivas ( $\Delta \underline{u})$, torna a função a ser minimizada descrita na forma:

A ponderação para os pesos dos erros

$$
J(\Delta u)=\underline{\hat{E}}^{T} \cdot Q \cdot \underline{\hat{E}}+\Delta \underline{u}^{T} \cdot R \cdot \Delta \underline{u}
$$

Em que: Onde $\mathrm{Q}$ e $\mathrm{R}$ são matrizes diagonais positivas semidefinidas, para ponderar os pesos de cada componente. A lei de

$$
\Delta u=\left(A^{T} \cdot Q \cdot A+R\right)^{-1} \cdot A^{T} \cdot Q \cdot \widehat{E}^{\prime}
$$

O ajuste dos parâmetros para o DMC não foi rigoroso, mantendo parâmetros semelhantes àqueles ajustados para as RNAs. As restrições para as predições da RNA inclui a faixa para a qual foi treinada $\left(980,4-1359,9^{\circ} \mathrm{F}\right)$, permitindo a extrapolação, na qual a rede neural apresenta uma ativação igual a 0 e 1 $\left(933,0-1407,3^{\circ} \mathrm{F}\right)$. A estratégia de controle por RNAs também inclui restrições nas ações de controle, impondo que esta variável seja mantida dentro de uma faixa de operação (Tabela 4).

Tabela 4 - Restrições para o controle preditivo baseado em RNAs.

\begin{tabular}{lc}
\hline & Restrições nas ações de controle \\
\hline $\mathrm{u}_{\text {mín }}$ & $74,71 \mathrm{~kg} / \mathrm{s}$ \\
$\mathrm{u}_{\text {máx }}$ & $107,39 \mathrm{~kg} / \mathrm{s}$ \\
$\Delta \mathrm{u}_{\text {máx }}$ & $1,26 \mathrm{~kg} / \mathrm{s}$ \\
\hline
\end{tabular}

A variável de controle escolhida é a temperatura do riser, manipulando a vazão de ar para o regenerador. Os valores dos parâmetros ajustados nos testes de controle são dados na Tabela 5, tanto para o caso servo, como para o caso regulador. 
Vivianni Marques Leite dos Santos

Tabela 5 - Valores dos parâmetros de ajuste para os controladores.

\begin{tabular}{lcc}
\hline Parâmetros de ajuste & Controle Baseado em RNAs & Controle DMC \\
\hline $\mathrm{H}$ & 10 & 10 \\
Alfa & 0,95 & 0,95 \\
$\mathrm{U}$ & 1 & 1 \\
Restrição na variável manipulada & $1 \times 10^{-6}$ & $1 \times 10^{-6}$ \\
Tempo de amostragem & $0,05 \mathrm{~h}(180 \mathrm{~s})$ & $0,05 \mathrm{~h}(180 \mathrm{~s})$ \\
Tempo de simulação & $15 \mathrm{~h}(54000 \mathrm{~s})$ & $15 \mathrm{~h}(54000 \mathrm{~s})$ \\
$\mathrm{N}$ & --- & 100 \\
\hline
\end{tabular}

\section{Problema servo}

Para o teste do controlador em um problema servo, o setpoint da temperatura do riser $(881,26 \mathrm{~K})$ foi aumentado, inicialmente para $923,49 \mathrm{~K}$ e em seguida reduzido para 839,06 K (Figura 10). O comportamento da variável de controle pode ser visualizado na Figura 10 (a) e os movimentos da variável manipulada (vazão de ar para o regenerador) na
Figura 10 (b).

Os resultados mostram que ambos os controladores conduzem a variável do processo para o novo setpoint, manipulando a vazão de ar com tempos de resposta bastante próximos. Dessa forma, os resultados mostram que o desempenho tanto do controlador DMC, como aquele baseado em RNAs, foi bastante satisfatório.

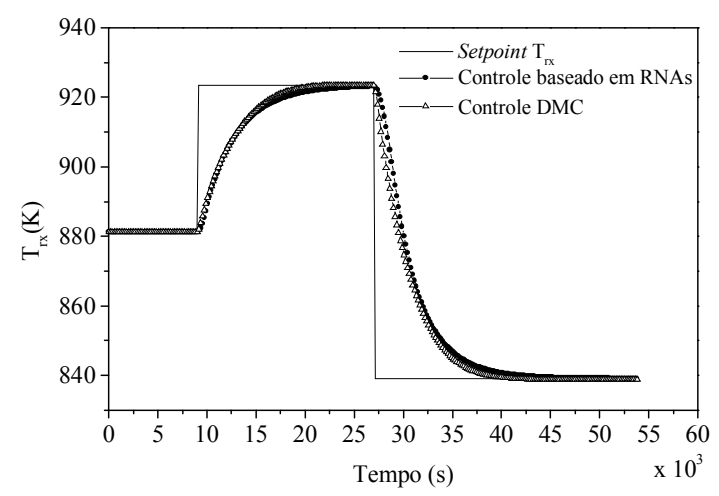

(a)

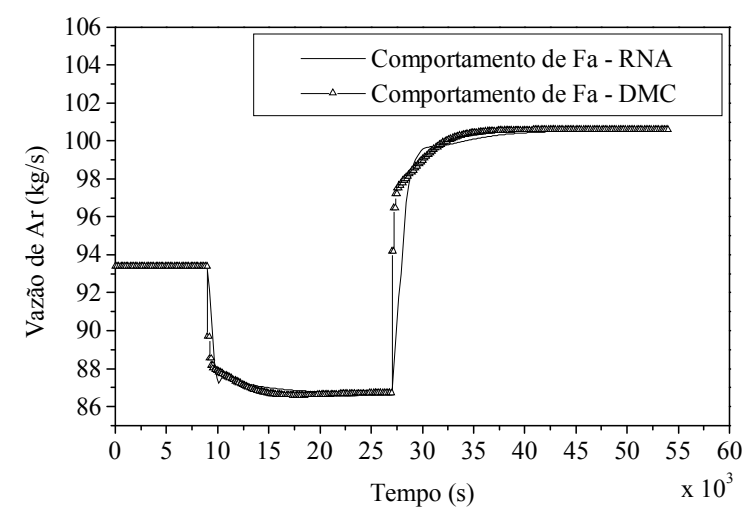

(b)

Figura 10: Problema Servo. Desempenho dos controladores para variações de 42,22 K ( $\mathrm{t}=2,5 \mathrm{~h}$ ou $\left.9 \times 10^{3} \mathrm{~s}\right)$ e $-42,22 \mathrm{~K}\left(\mathrm{t}=7,5 \mathrm{~h}\right.$ ou $\left.27 \times 10^{3} \mathrm{~s}\right)$ no setpoint inicial da temperatura do riser (a) e comportamento da variável manipulada (b).

\section{Problema regulador}

Para a análise do desempenho dos controladores obtidos neste trabalho, a variável de perturbação escolhida foi a temperatura do ar para o regenerador, uma vez que, tem forte interação com as temperaturas do riser e do regenerador. Para uma perturbação de 15,27 K $\left(\mathrm{t}=9 \times 10^{3}\right.$ s) e $-15,27 \quad \mathrm{~K} \quad\left(\mathrm{t}=27 \times 10^{3}\right.$ s $)$ na temperatura inicial de $305,37 \mathrm{~K}$, manipulando a vazão de ar para o regenerador, o desempenho dos controladores para a temperatura do riser é 
mostrado na Figura 11 (a) e o comportamento da variável manipulada é apresentado na mesma figura à direita. Neste caso, o controle preditivo baseado em RNAs se mostrou mais

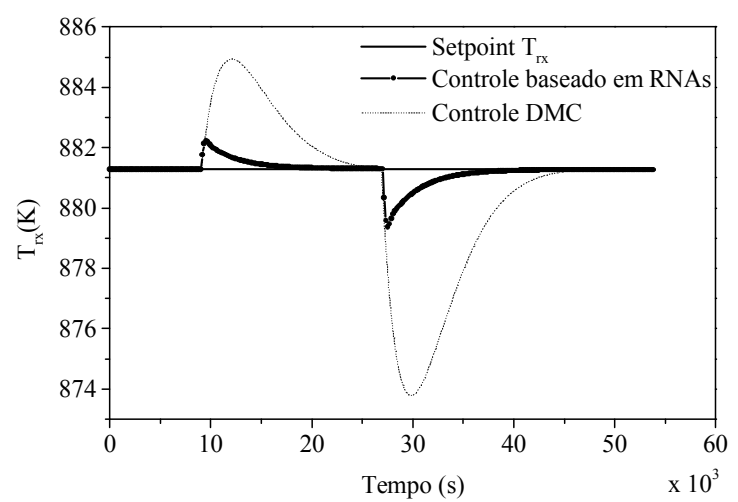

(a) eficiente, não permitindo atingir picos de temperatura, além de retornar mais rapidamente ao valor desejado (setpoint) da variável controlada.

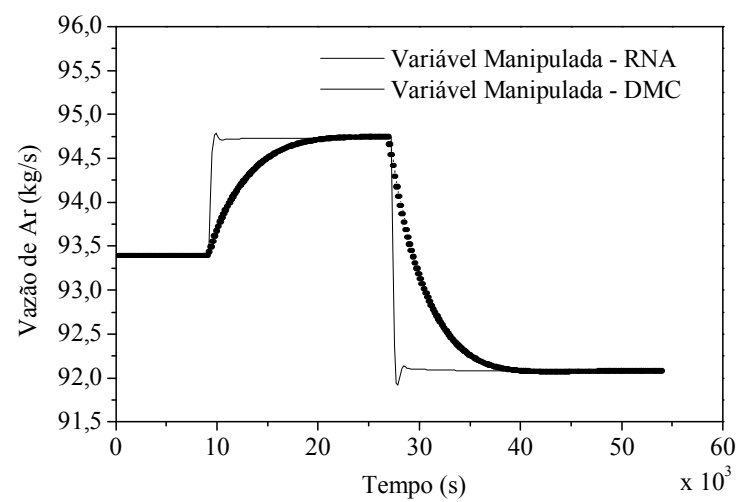

(b)

Figura 11: Problema Regulador. Desempenho dos controladores para degraus de $+15,27 \mathrm{~K}$ em $\mathrm{t}=$ $9 \times 10^{3} \mathrm{~s}(2,5 \mathrm{~h}) \mathrm{s} \mathrm{e}-15,27 \mathrm{~K} \mathrm{em} \mathrm{t}=27 \times 10^{3} \mathrm{~s}(7,5 \mathrm{~h})$ na temperatura do ar para o regenerador, correspondente ao estado estacionário inicial (a) e o comportamento da variável manipulada (b).

O controle MISO (Multiple Input Single Output) baseado em redes neurais da temperatura do riser fez com que a temperatura do regenerador retornasse ao setpoint rapidamente ( $\sim 6$ segundos), sem variação maior que 1,0 K, ao passo que o controle DMC também conduziu à temperatura do regenerador para o setpoint, entretanto após variação maior da temperatura $(\sim 4,7 \mathrm{~K})$ e do tempo $(\sim 10$ segundos) (Figura 12).

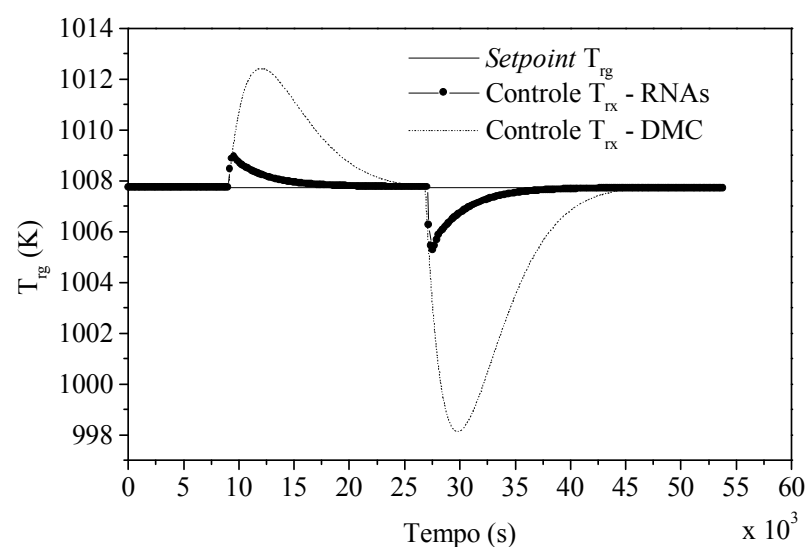

Figura 12: Problema Regulador. Comportamento da temperatura do regenerador, com atuação de controle para a temperatura do riser.

Dessa forma, foi verificado que para o Caso Regulador, o controle da temperatura do riser, mantém a temperatura do regenerador no seu setpoint. 


\section{Conclusões}

Os resultados da identificação mostram que as RNAs do tipo multicamadas foram capazes de predizer o comportamento das variáveis principais do processo. $\mathrm{O}$ comportamento não linear foi identificado pelas RNAs, que também superaram desafios ainda maiores, predizendo os estados estacionários do modelo razoavelmente bem.

A bifurcação dinâmica para as concentrações de coque nos catalisadores determinaram a região de operação possível (pontos estáveis e instáveis) do ponto de vista da estabilidade operacional.

O controle MISO para a temperatura do riser mostrou-se bastante eficiente. No caso regulador, a temperatura voltou muito rapidamente para o estado estacionário estável de operação, necessitando de uma ação de controle enérgica. $\mathrm{O}$ controle baseado no algoritmo DMC também foi satisfatório, embora permita atingir picos maiores de temperatura, antes de retornar ao setpoint. Dessa forma, no caso servo o desempenho foi o mesmo em ambos os controladores e para o caso regulador foi obtida maior eficiência do controle baseado em RNAs. Logo, RNAs podem ser utilizadas para estimar variáveis do processo de craqueamento catalítico do petróleo e dessa forma permitir estimativas online na unidade.

Como perspectiva deste trabalho, podese testar outros ajustes no controle preditivo baseado em RNAs para os dois modelos e no controlador baseado no algoritmo DMC. Também pode ser testada a inclusão de restrições para o DMC, utilizando o algoritmo LDMC (Linear Dynamic Model Control) ou o QDMC (Quadratic Dynamic Matrix).

\section{Nomenclatura}

\section{Modelo}

Símbolos Latinos:

$a_{c b}$ - Fator de frequência para a reação de queima de coque $\left(\mathrm{s}^{-1}\right)$

$\mathrm{a}_{\mathrm{cc}}$ - Constante catalítica de formação de coque (kg coque/ kg catalisador)
$\mathrm{C}_{\mathrm{pc}}-$ Calor específico do catalisador $(\mathrm{J} / \mathrm{kg}$ catalis. $\mathrm{K}$ )

$\mathrm{C}_{\mathrm{pf}}-$ Calor específica da alimentação $(\mathrm{J} / \mathrm{kg}$ carga $\mathrm{K}$ )

$\mathrm{C}_{\mathrm{rg}}-$ Conteúdo de coque no catalisador regenerado ( $\mathrm{kg}$ coque $/ \mathrm{kg}$ catalisador)

$\mathrm{C}_{\mathrm{sc}}$ - Conteúdo de coque no catalisador gasto ( $\mathrm{kg}$ coque/ $\mathrm{kg}$ catalisador)

$\mathrm{E}_{\mathrm{cb}}$ - Energia de ativação para a reação de queima de coque $(\mathrm{J} / \mathrm{mol})$

$\mathrm{E}_{\mathrm{cc}}$ - Energia de ativação para a reação de formação de coque catalítico $(\mathrm{J} / \mathrm{mol})$

$\mathrm{F}_{\mathrm{a}}$ - Vazão de ar $(\mathrm{kg} / \mathrm{s})$

$\mathrm{F}_{\mathrm{c}}$ - Taxa de circulação de catalisador $(\mathrm{kg} / \mathrm{s})$

$\mathrm{F}_{\mathrm{g}}$ - Vazão de gases no regenerador $(\mathrm{kg} / \mathrm{s})$

$\mathrm{F}_{\mathrm{t}}$ - Vazão da alimentação combinada $(\mathrm{kg} / \mathrm{s})$

$\mathrm{H}_{\mathrm{rg}}$ - "Hold up" de catalisador no regenerador

$(\mathrm{kg})$

$\mathrm{H}_{\mathrm{rx}}$ - "Hold up" de catalisador no reator $(\mathrm{kg})$

$\mathrm{M}_{\mathrm{c}}$ - Peso molar de coque $(\mathrm{kg} / \mathrm{mol})$

$\mathrm{M}_{\mathrm{g}}$ - Peso molar médio dos gases no regenerador $(\mathrm{kg} / \mathrm{mol})$

$\mathrm{Q}_{\mathrm{cb}}$ - Calor de combustão do coque $(\mathrm{J} / \mathrm{kg}$ coque)

$\mathrm{Q}_{1}$ - Energia de ativação para a reação de craqueamento $(\mathrm{J} / \mathrm{mol})$

$\mathrm{Q}_{3}$ - Energia de ativação para desativação $(\mathrm{J} / \mathrm{mol})$

$\mathrm{R}$ - Constante dos gases $(\mathrm{J} / \mathrm{mol} \mathrm{K})$

$\mathrm{R}_{\mathrm{cb}}$ - Taxa de queima de coque total $(\mathrm{kg}$ coque $/ \mathrm{s}$ )

$\mathrm{R}_{\mathrm{cf}}$ - Taxa de formação de coque total $(\mathrm{kg}$ coque/s)

$\mathrm{T}_{\mathrm{a}}$ - Temperatura do ar $(\mathrm{K})$

$\mathrm{T}_{\mathrm{f}}-$ Temperatura da alimentação $(\mathrm{K})$

$\mathrm{T}_{\text {mix }}$ - Temperatura da mistura no riser $(\mathrm{K})$

$\mathrm{T}_{\mathrm{r}}$ - Temperatura de reação $(\mathrm{K})$

$\mathrm{T}_{\mathrm{rg}}$ - Temperatura do leito do regenerador $(\mathrm{K})$

$\mathrm{T}_{\mathrm{rx}}-$ Temperatura do leito do reator $(\mathrm{K})$

$t_{c}$ - tempo de residência do catalisador (s)

Símbolos Gregos:

$\alpha_{\mathrm{CO}} \quad-$ Razão estequiométrica de carbono/oxigênio

$\Delta \mathrm{H}_{\mathrm{cr}}$ - Calor da reação de craqueamento $(\mathrm{J} / \mathrm{kg}$ craqueada)

$\lambda_{0}-$ Calor de vaporização do óleo (J/kg carga) 
Vivianni Marques Leite dos Santos

Redes neurais e controle preditivo DMC

Símbolos Latinos:

f - Função de ativação

H - Horizonte de predição

$\mathrm{J}$ - função objetivo para controle

ta-tempo de amostragem

$\mathrm{m}$ - parâmetro relacionado a ordem do modelo

de identificação

$\mathrm{n}$ - parâmetro relacionado a ordem do modelo

de identificação

$\mathrm{n}_{\mathrm{h}}-$ Número de neurônios na camada escondida

$\mathrm{r}$ - Valor do setpoint

$\mathrm{R}$ - Matriz definida positiva, acrescentada para restringir os movimentos excessivos na variável manipulada $\Delta \mathrm{u}$

$\mathrm{sp}$ - setpoint

$\mathrm{T}$ - Horizonte do modelo

$\mathrm{T}_{\mathrm{a}}$ - Período de amostragem

U- horizonte de cotrole

u - variável manipulada (ação de controle)

y- variável de saída controlada

$\mathrm{y}^{\mathrm{a}}$ - valor desejado de y no instante de tempo atual

$\mathrm{y}_{\mathrm{o}}$ - Valor de y no estado estacionário inicial

$y_{i}$ - Valor da variável controlada, em cada instante de tempo

$\mathrm{y}^{*}$ - Predição corrigida de y em cada instante de tempo

$\widehat{y}_{\text {- saída predita pelo modelo }}$

$\hat{y}_{d}$ - predição corrigida do modelo

$y_{\min }, y_{\text {máx }}$ - restrições de posição nas variáveis controladas

$u_{\min }, u_{\text {máx }}$ - restrições de posição nas variáveis manipuladas

$y_{x p}$ - trajetória de referência

Símbolos Gregos:

$\alpha$ - parâmetro ajustável para a trajetória de referência

$\Delta \mathrm{u}$ - incremento na variável manipulada

$\Delta \mathrm{u}_{\text {máx }}$ - restrição de velocidade na variável manipulada

$\lambda$ - restrição nos incrementos das ações de controle

\section{Referências}

ALGHAZZAWI A., LENNOX B. - Model predictive control monitoring using multivariate statistics. Journal of Process Control 19, 314-327, 2009.

ARAÚJO A. S., M. G. F. Rodriguez. Desenvolvimento de Catalisadores Zeolíticos Destinados à Reforma do Metano. Tese de Doutorado. Área de Concentração: Operações e Processos, Centro de Ciências e Tecnologia, Universidade Federal de Campina Grande. 2006.

ARMOR J. N. - A history of industrial catalysis. Catalysis Today, 2010.

DARAOUI N., DUFOUR P., HAMMOURI H., HOTTOT A. - Model predictive control during the primary drying stage of lyophilisation. Control Engineering Practice. 18, 483-494, 2010.

DE SOUZA JR. M. B. - Redes Neuronais Multicamadas Aplicadas a Modelagem e Controle de Processos Químicos. Tese de Doutorado. COPPE/PEQ/UFRJ. Rio de Janeiro, 1993.

DOEDEL E. J., FAIRGRIEVE T. F., SANDSTEDE B., CHAMPNEYS A. R., KUZNETSOV Y. A., WANG X. - Continuation and Bifurcation Software for Ordinary Differential Equations. Manual AUTO97. March 29, 1998.

FIEDLER FERRARA N. \& PRADO C. P. C. Caos - Uma Introdução. Editora Edgard Blircher Ltda. S. Paulo - SP. 1995.

FILETI A. M. F. - Controle Avançado de Processos: Adaptativo, Preditivo e Fuzzy. Modelagem Matemática via Redes Neurais. Aplicações em Processos Químicos. Material especialmente elaborado para curso ministrado na Escola Politécnica da Universidade Federal da Bahia. Julho, 1998.

LEE W. \& KUGELMAN A. M. - Number of steady-state operating points and local stability of open-loop fluid catalytic cracker. Ind. Eng. Chem. Pocess Des. Dev., 12, 197-204, 1973.

LEONARD J. \& KRAMER, M. A., Improvement of the Backpropagation Algorithm for Training Neural Networks, Computers chem. Engng., 14, $\mathrm{N}^{\mathrm{0}} 3,337-341$, 1990.

NARENDRA, $\quad$ K. S.

AND 
Vivianni Marques Leite dos Santos

PARTHASARATHY, K. "Identification and Control of Dynamical Systems Using Neural Networks," IEEE Trans. Neural Networks, 1(1), 4-27, 1999.

RUMELHART D. E. \& McCLELLAND J. L. Parallel Distributed Processing: Explorations in the Microstructure of Cognition. 1, Foundations the Mit Press. Cambridge. 1986.

SCATTOLINI R. - Architectures for distributed and hierarchical Model Predictive Control - A review. Journal of Process Control.19, 723-
731, 2009.

STEWART B. T., VENKAT A. N., RAWLINGS, J. B., WRIGHT, S. J., PANNOCCHIA G. - Cooperative distributed model predictive control. Systems \& Control Letters 59, 460 469, 2010.

SU H., MCAVOY T. J., WERBOS P. - Longterm Predictions of Chemical Processes Using Recorrent Neural Neworks: a Parallel Training Approach. Ind. Eng. Chem. Res., 31, 13381352, 1992. 